\title{
PREVALENNCIA E NOTIFICAÇÕES DE ACIDENTES DE TRABALHO COM EXPOSIÇÃO A MATERIAL BIOLÓGICO NA ODONTOLOGIA
}

\section{Prevalence and work accident notification with exposure to biological material in Dentistry}

Sumaia Austregésilo Nogueira - Cirurgiã-Dentista. Mestre em Saúde Coletiva pela Universidade Federal do Rio Grande do Norte-UFRN. E-mail: sumaia.nogueira@hotmail.com

Bruna Katherine Guimarães Carvalho - Cirurgiã-Dentista pela UFRN. Ex Bolsista de Iniciação Científica do CNPq. E-mail: brunaguimaraes1919@gmail.com

Andressa da Rocha Medeiros • Cirurgiã-Dentista pela UFRN. Ex Bolsista de Iniciação Científica da PROPESQ-UFRN. E-mail: andressa_rmedeiros@hotmail.com

Shênia Eliane do Rego Carneiro • Cirurgiã-Dentista pela UFRN. Ex Bolsista de Iniciação Científica da PROPESQ-UFRN. E-mail: sheniacarneiro@ig.com.br

Georgia Costa de Araújo Souza - Cirurgiã-Dentista. Doutora em Saúde Coletiva pela UFRN. Professora do Curso de Odontologia da Universidade do Estado do Rio Grande do Norte-UERN. E-mail: georgia_odonto@yahoo.com.br

Autora responsável pela correspondência:

Sumaia Austregésilo Nogueira - E-mail: sumaia.nogueira@hotmail.com 


\section{RESUMO}

Introdução Os acidentes de trabalho, principalmente os que envolvem exposição a material biológico, podem ocorrer no dia-a-dia do cirurgião-dentista e dos auxiliares em saúde bucal, sendo um sério risco ocupacional. Conhecendo sua real magnitude, é possível que estes acidentes não estejam sendo devidamente registrados.

Objetivo: Conhecer a prevalência de comunicações de acidentes de trabalho típicos com exposição a material biológico na equipe de saúde bucal da Rede básica de saúde nos anos 2008 a 2009 na cidade de Natal-RN. Metodologia: Neste estudo descritivo, do tipo transversal foram utilizadas na coleta de dados as comunicações de acidentes de trabalho, cadastradas no "Núcleo de Agravos Notificáveis da Secretaria Municipal de Saúde de Natal-RN" além de um questionário semiestruturado. Resultados: Dos 54 acidentes perfuro-cortantes registrados, 70,37\% $(n=38)$ aconteceram em indivíduos do sexo feminino, 53,70\% ( $n=29)$ em indivíduos com idades entre 20 e 30 anos, e a categoria que mais registrou acidentes foram os cirurgiões-dentistas $(n=28$; $51,85 \%$ ). As lesões percutâneas foram as mais prevalentes e o sangue foi o material biológico envolvido na maior parte destes acidentes. Os procedimentos clínicos relacionados a periodontia e cirurgia foram os mais citados pelos profissionais e as agulhas com lúmen o instrumento mais relatado como causador dos acidentes. Para os auxiliares em saúde bucal a lavagem ou descarte inadequado de material esteve relacionada a 84,6\% dos acidentes ocorridos. Dos profissionais acidentados, 66,66\% apresentavam cobertura vacinal completa. Conclusões: Percebe-se subnotificação de acidentes, considerando-se que o número de profissionais existentes na cidade de Natal atuantes no período estudado é potencialmente maior que o número de acidentes registrados. Sugere-se sensibilização dos profissionais em relação à importância do registro de acidentes de trabalho, para controle dos acidentes ocupacionais e proteção à saúde do trabalhador.

Palavras-chave: Risco ocupacional; Acidente de trabalho; Saúde do trabalhador. 


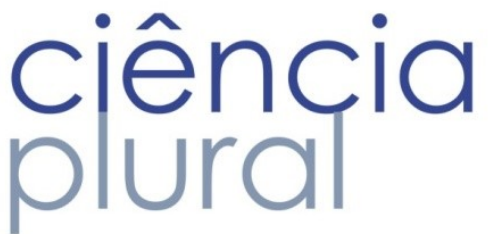

\section{ABSTRACT}

Introduction: Work accidents, especially those involving exposure to biological material, can occur in day-today of dentists and oral health assistants, and its a serious occupational hazard. Knowing their real magnitude, it is possible that these accidents are not being properly registered. Objective: Know the prevalence of accidents notifications with exposure to biological material in the oral health team, in the primary care network in the years 2008 to 2009 the city of Natal-RN. Methods: In this descriptive, cross-sectional research, were used in data collection the work accidents communications, registered in the "Núcleo de Agravos Notificáveis da Secretaria Municipal de Saúde de Natal-RN" plus a semi structured questionnaire.. Results: Of the 54 accidents recorded needlestick injuries, $70.37 \%(n=38)$ occurred with females, $53.70 \%(n=29)$ aged between 20 and 30 years, and the category that registered more accidents were the dentists $(n=28 ; 51.85 \%)$. The percutaneous injuries were the most prevalent and the blood biological material was involved in the majority of these accidents. The clinical procedures of periodontics and surgery were the most commonly mentioned by professionals and lumen needles were the most reported instrument as the cause of accidents. For oral health assistants, washing or improper disposal of materials was related to $84.6 \%$ of accidents. About the professionals who have suffered accidents, $66.66 \%$ had complete vaccination coverage. Conclusions: It is noticed there is underreporting of accidents, considering that the number of active professionals in Natal during the study period is potentially greater than the number of registered accidents, It is suggested the awareness of professionals about the importance of registration of workplace accidents, for control of occupational accidents and protection of worker's health.

Keywords: occupational risk; work accident; worker's health. 


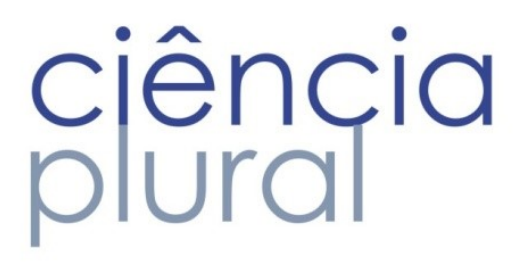

Introdução

Os acidentes de trabalho são acontecimentos não planejados que ocorrem no exercício laboral provocando danos ou agravos à saúde, como lesões corporais ou perturbações funcionais que causem a morte, a perda ou redução da capacidade para o trabalho de modo permanente ou temporário ${ }^{1,2}$. São tradicionalmente classificados em acidentes típicos (os ocorridos no ambiente de trabalho e/ ou durante a jornada de trabalho que corresponde aqueles cuja Comunicação de Acidentes do Trabalho - CAT foi cadastrada no Instituto Nacional de Seguridade Social-INSS) e acidentes de trajeto (os ocorridos no trajeto da residência para o trabalho e do trabalho para a residência) $)^{1,2}$.

Por sua vez, a doença ocupacional ou a doença do trabalho são equiparadas a acidente de trabalho, conforme previsto no art. 20 da lei 8.213 , de 1991. Entende-se como a doença profissional ou do trabalho aquela produzida ou desencadeada pelo exercício de trabalho peculiar a determinada atividade, assim como acidente ou doença ligada ao trabalho, que embora não sejam provocados diretamente pelo trabalho, com ele tem relação, doença proveniente de contaminação acidental do empregado no exercício de sua atividade e no deslocamento no itinerário - percurso de casa para o trabalho e vice-versa1.

Portanto, os acidentes do trabalho (AT) são o maior agravo à saúde dos trabalhadores, tornando-se, assim, um importante problema de saúde pública, tanto em países desenvolvidos, quanto em países em desenvolvimento. São uma fonte de preocupações para as instituições e os trabalhadores, constituindo-se tema de grande importância também para pesquisas relacionadas à saúde do trabalhador no Brasil e no mundo. A Lei n. 8.213, de 24 de julho de 1991, que consolida a legislação que dispõe sobre os Planos de Benefícios e Custeio da Previdência Social e sobre a organização da Seguridade Social e dá outras providências, define acidente de trabalho como aquele que ocorre pelo exercício do trabalho, a serviço da empresa, provocando lesão corporal ou perturbação funcional que cause a morte, a perda ou redução da capacidade para o trabalho permanente ou temporária ${ }^{1,2}$. Os trabalhadores da área de saúde ficam expostos a inúmeros acidentes de trabalho devido aos procedimentos realizados em seu dia a dia. Desses, sabe-se que os cirurgiões-dentistas são profissionais dos que estão mais expostos a esses acidentes, principalmente aqueles causados por materiais perfuro-cortantes e fluidos biológicos ${ }^{1}$.

Estes acidentes podem ocorrer na rotina diária da equipe de saúde bucal, principalmente os que envolvem exposição a material biológico, e frequentemente se devem à dinâmica dos procedimentos realizados na prática clínica, que envolve o manuseio de instrumentos perfuro-cortantes. Porém, a literatura sobre saúde ocupacional indica que diversos fatores estão associados aos acidentes, como o estresse ao qual os profissionais estão expostos, bem como falta de atenção, pressa e nervosismo ${ }^{3,4}$.

O risco biológico está definitivamente presente entre os trabalhadores da saúde bucal, e por ocasião de acidentes de trabalho, esses profissionais ficam expostos a material biológico e várias doenças podem ser transmitidas como hepatites, sífilis e síndrome da imunodeficiência adquirida (SIDA). Os acidentes de trabalho são bastante frequentes em odontologia, já que existe uma manipulação constante de objetos pontiagudos e motores de alta rotação. Os instrumentos podem causar ferimentos e abrasões nas mãos dos dentistas, e, ainda, fragmentos dentários ou líquidos podem ser projetados com consequente risco de lesão cutânea ou 


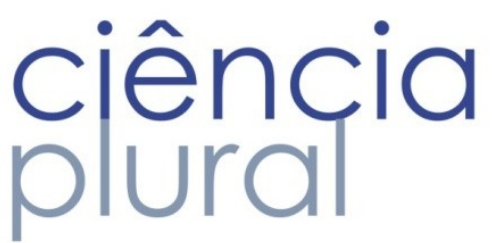

ocular. A equipe odontológica está, então, sob constante risco de se contaminar por esses agentes infecciosos 4,5 .

As consequências de uma exposição ocupacional a patógenos veiculados pelo sangue vão além do comprometimento físico a curto ou longo prazo e podem afetar outros aspectos da saúde profissional, tais como: controle emocional, social e, até, financeiro. $\mathrm{O}$ acidente envolvendo material biológico potencialmente contaminado pode trazer repercussões psicossociais ao profissional acidentado, provocando mudanças nas relações sociais, familiares e de trabalho ${ }^{5}$.

A discussão sobre os riscos ocupacionais aos quais estão submetidos os profissionais de saúde e a possibilidade de adoecimento em decorrência destes é relativamente recente. A partir do surgimento da SIDA, houve maior utilização de medidas de precaução universal no intuito de prevenir contaminação e acidentes ocupacionais 6 .

O número de acidentes e doenças ocupacionais ocorridos com profissionais da saúde vem aumentando devido a fatores, como excesso de carga horária de trabalho, estresse, uso incorreto da biossegurança, estado emocional dos pacientes e/ou profissionais durante 0 atendimento, acrescentando-se que as condições de trabalho dos cirurgiões-dentistas e auxiliares de consultório odontológico fazem com que eles estejam expostos a uma grande variedade de micro-organismos presentes, especificamente, no sangue, na saliva e nas vias aéreas respiratórias dos pacientes ${ }^{6}$.

Dados referentes a acidentes de trabalho em odontologia ainda são escassos na literatura. Um dos aspectos amplamente relatados na literatura é a ausência de Sistemas de Informação (SI) que permitam estimar e acompanhar o real impacto desses acidentes sobre a saúde do trabalhador. A inexistência de SI destinados à captação dos acidentes de trabalho ocorridos fora da população previdenciária coberta pelo seguro de acidente de trabalho (SAT), que é a maioria da população trabalhadora, é evidente. Há também evidências de casos de não-diagnóstico e/ou não-registro de acidentes, principalmente no que se refere aos casos de doenças relacionadas ao trabalho ${ }^{7}$.

A prática da Odontologia, por abranger uma grande variedade de procedimentos com diferentes níveis de complexidade, geralmente implica em contato com secreções da cavidade oral, a exemplo de saliva, sangue e outros tipos de secreções, como as das vias aéreas superiores, além de aerossóis, sendo fator de risco para a transmissão de infecções entre profissionais e pacientes. 0 registro de acidentes constitui-se em importante instrumento para planejamento e verificação da eficácia de medidas de prevenção. A notificação aos órgãos de Vigilância à Saúde do Trabalhador garante dados para pesquisas sobre acidentes, possibilitando ainda orientar as políticas públicas, visando à diminuição destas ocorrências ${ }^{1,8}$.

A literatura revela que, muitas vezes, o diagnóstico e/ ou o registro não ocorrem, caracterizando a subnotificação e impedindo o conhecimento da real magnitude dos acidentes ocorridos e suas consequências na saúde do trabalhador. Assim, podem ocorrer grandes prejuízos em longo prazo, deixando o trabalhador desamparado e sem condições de comprovar qualquer dano à sua saúde ocasionado ou relacionado ao acidente ${ }^{9}$. 


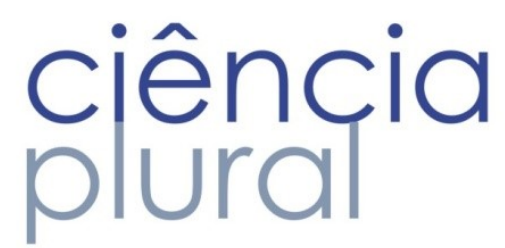

Os cuidados imediatos após as lesões percutâneas se resumem à lavagem exaustiva do local exposto com água e sabão. Nas exposições que atingem as mucosas, deve ser realizada lavagem exaustiva com água ou solução salina fisiológica. Pacientes e profissionais devem ser submetidos a testes sorológicos para investigar possível infecção prévia por HIV ou HBV, e, caso indicada (paciente-fonte com sorologia positiva ou desconhecida para tais vírus), a quimioprofilaxia deve ser iniciada dentro das primeiras 24 a 48 horas após a exposição. É importante que os profissionais que sofreram exposições ocupacionais sejam adequadamente atendidos e orientados por um profissional que esteja atento aos aspectos psicossociais relacionados ao acidente de trabalho, como a síndrome da desordem pós-traumática com reações de medo, angústia, ansiedade e depressão $0^{10,11}$.

Não há dados suficientes na literatura que comprovem o registro através de Sistema de Informações dos acidentes de trabalho ocorridos na equipe de saúde bucal. É possível que os acidentes não sejam devidamente registrados considerando sua real magnitude. Desta forma, torna-se importante ressaltar a relevância não apenas da pesquisa da prevalência de acidentes de trabalho em profissionais de saúde bucal, mas também da existência ou não de registros destes acidentes.

Considerando a possível ocorrência de acidentes ocupacionais envolvendo exposição a material biológico na equipe de saúde bucal e suas potenciais consequências para a saúde destes profissionais, concomitantemente com a hipótese de subnotificações destes acidentes aos órgãos de Vigilância de Saúde do Trabalhador, ressalta-se a relevância e atualidade deste tema. Assim ao estudar os acidentes ocupacionais notificados entre a equipe odontológica contribui-se com informações que possibilitarão maiores reflexões sobre a saúde do trabalhador e desta forma segurança e qualidade de vida podem ser repensadas e incorporadas pelas instituições e pelos profissionais de saúde no seu exercício laboral.

\section{Materiais e Métodos}

Tratou-se de um estudo descritivo, transversal. A população do estudo foi constituída de cirurgiõesdentistas e auxiliares em saúde bucal das unidades de saúde da Secretaria Municipal de Saúde da cidade de Natal - RN que sofreram acidente de trabalho no período 2008-2009.

Os critérios de inclusão dos sujeitos na pesquisa foram ser cirurgião-dentista ou auxiliar em saúde bucal em exercício nas unidades de saúde da Secretaria Municipal de Saúde de Natal. Profissionais em qualquer tipo de licença, férias, os não localizados ou que não exerceram atividade clínica no período estudado foram excluídos.

Foram utilizados dois instrumentos de coleta de dados: questionário autoaplicável semiestruturado, previamente testado, distribuído a cirurgiões-dentistas e auxiliares em saúde bucal na unidade de saúde da sua lotação e as Comunicações de Acidentes de Trabalho - CAT, registradas no Núcleo de Acidentes NotificáveisNAN da Secretaria Municipal de Saúde da cidade de Natal-RN. 


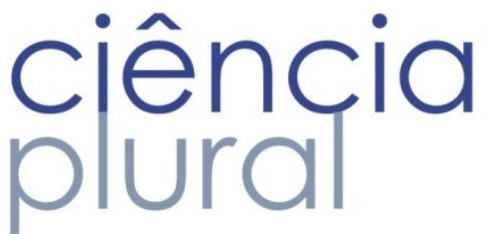

Dados sócio demográficos como sexo, idade, tempo de formado e se 0 trabalhador fez algum treinamento em biossegurança foram também coletados. Em relação às circunstâncias dos acidentes foi pesquisado se 0 trabalhador sofreu algum acidente de trabalho naquela instituição no período estudado, 0 material biológico envolvido no acidente, o tipo de procedimento que estava sendo realizado, a possível causa e objeto causador do acidente, o período do dia no momento do acidente, a quantidade de horas trabalhadas no dia do ocorrido, qual a principal parte do corpo envolvido no acidente, uso de equipamento de proteção individual e ainda, a doença que mais preocupa o trabalhador em caso de exposição a material biológico. Foram levados em consideração apenas os acidentes ocorridos naquela instituição e nos casos de ocorrência de mais de um acidente com o mesmo profissional, foi levado em consideração apenas o último para fins de estatística.

Em relação à notificação dos acidentes foi pesquisado o conhecimento da importância da notificação dos acidentes de trabalho. $O$ registro das notificações dos acidentes de trabalho com exposição a material biológico em cirurgiões-dentistas e auxiliares em saúde bucal foi pesquisada no Núcleo de Agravos Notificáveis da Secretaria Municipal de Saúde de Natal - RN através de análise das Comunicações de Acidente de TrabalhoCAT disponíveis no local.

Para minimizar o viés de memória, foi delimitado o tempo de ocorrência dos acidentes aos dois anos anteriores à coleta de dados. Os dados coletados através dos questionários foram inseridos em um Banco de Dados e analisados com o auxílio do software SPSS versão 17.0 e foram interpretados de forma descritiva através da frequência absoluta e relativa. 0 Termo de Consentimento Livre e Esclarecido foi apresentando a cada sujeito antes do início da pesquisa, e o estudo foi autorizado pelo Comitê de Ética do Hospital Universitário Onofre Lopes-UFRN através do parecer $n^{0} 361 / 09$.

\section{Resultados}

Os questionários foram entregues a 109 cirurgiões-dentistas e 100 auxiliares em saúde bucal no seu ambiente de trabalho, dos quais 82 e 73 profissionais responderam respectivamente. A idade média dos cirurgiões-dentistas foi de 50 anos $( \pm 7,3)$ e dos auxiliares em saúde bucal foi de 46 anos $( \pm 9,11)$, sendo que $32,9 \%(n=27)$ dos cirurgiões-dentistas são do sexo masculino enquanto que apenas $1,4 \%(n=1)$ dos auxiliares em saúde bucal é do sexo masculino. As CAT segundo as variáveis demográficas relacionadas à população estudada estão agrupadas na Tabela 1. 
Tabela 1: Comunicações de Acidentes de Trabalho com exposição a material biológico, cadastradas no Núcleo de Acidentes Notificáveis segundo as variáveis sexo, idade e categoria profissional em Odontologia. Natal-RN. 2010.

\begin{tabular}{|c|c|c|c|c|c|c|c|}
\hline \multirow{3}{*}{\multicolumn{2}{|c|}{$\begin{array}{c}\text { COMUNICAÇÃO DE ACIDENTE } \\
\text { DE TRABALHO - CAT }\end{array}$}} & \multicolumn{2}{|c|}{2008} & \multicolumn{2}{|c|}{2009} & \multicolumn{2}{|c|}{2008 e 2009} \\
\hline & & \multirow{2}{*}{$\frac{\mathbf{N}}{27}$} & \multirow{2}{*}{$\begin{array}{c}\% \\
100\end{array}$} & \multirow{2}{*}{$\frac{\mathbf{N}}{27}$} & \multirow{2}{*}{$\begin{array}{c}\% \\
100\end{array}$} & \multirow{2}{*}{$\begin{array}{l}\mathbf{N} \\
54\end{array}$} & \multirow{2}{*}{$\begin{array}{c}\% \\
100\end{array}$} \\
\hline & & & & & & & \\
\hline \multirow[t]{2}{*}{ Sexo } & Masculino & 9 & 33,33 & 7 & 25,92 & 16 & 29,62 \\
\hline & Feminino & 18 & 66,66 & 20 & 74,07 & 38 & 70,37 \\
\hline \multirow[t]{5}{*}{ Idade } & De 20 a 30 anos & 14 & 51,85 & 15 & 55,55 & 29 & 53,70 \\
\hline & De 31 a 40 anos & 7 & 25,92 & 8 & 29,62 & 15 & 27,77 \\
\hline & De 41 a 50 anos & 3 & 11,11 & 1 & 3,70 & 4 & 7,40 \\
\hline & Mais de 50 anos & 3 & 11,11 & 0 & 0 & 3 & 5,55 \\
\hline & Ignorado & 0 & 0 & 3 & 11,11 & 3 & 11,11 \\
\hline \multirow[t]{4}{*}{ Categoria } & Cirurgião-Dentista & 16 & 59,25 & 12 & 44,44 & 28 & 51,85 \\
\hline & Estudante & 2 & 7,40 & 4 & 14,80 & 6 & 11,11 \\
\hline & ASB & 8 & 29,62 & 11 & 40,74 & 19 & 35,18 \\
\hline & Auxiliar de Prótese & 1 & 3,70 & 0 & 0 & 1 & 1,85 \\
\hline
\end{tabular}

A média de tempo de formado de cirurgiões-dentistas foi de 26,86 anos $( \pm 5,92)$ enquanto a média de atuação dos auxiliares em saúde bucal na profissão foi de 18,61 anos $( \pm 8,95)$. Não houve cirurgiões-dentistas graduados há menos de 7 anos e apenas um auxiliar em saúde bucal $(1,36 \%)$ apresentou tempo de formação superior a 31 anos.

Dos cirurgiões-dentistas 12,8\% e dos auxiliares em saúde bucal 18,3\% sofreram acidentes de trabalho com exposição a material biológico. Os agentes causadores dos acidentes ocorridos na população estudada estão listados na Tabela 2. Todos os acidentes relatados pelos cirurgiões-dentistas envolveram exposição a sangue, e o material biológico, ao qual os auxiliares em saúde bucal foram mais expostos foi sangue (38,5\%), seguido da categoria outros (ex: água contendo instrumental sujo) $(30,8 \%)$. Procedimentos relacionados a cirurgia e periodontia foram mais relacionados aos acidentes ocorridos em cirurgiões-dentistas (40\%), enquanto que a lavagem ou descarte inadequado de material esteve relacionada a $84,6 \%$ dos acidentes ocorridos em auxiliares em saúde bucal. 


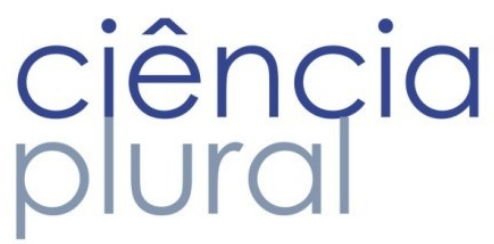

Tabela 2: Agentes causadores dos acidentes de trabalho com exposição a material biológico em Odontologia. Natal-RN. 2010.

\begin{tabular}{lllll}
\hline $\begin{array}{l}\text { Agentes causadores dos } \\
\text { acidentes de trabalho }\end{array}$ & $\mathbf{2 0 0 8}$ & & $\mathbf{2 0 0 9}$ & \\
& $\mathbf{N}$ & $\mathbf{\%}$ & $\mathbf{N}$ & $\mathbf{\%}$ \\
\hline Agulha com lúmen & 12 & 44,44 & 9 & 33,33 \\
Agulha sem lúmen & 0 & 0 & 3 & 11,11 \\
Lâmina/lanceta & 1 & 3,70 & 1 & 3,70 \\
Outros & 8 & 29,62 & 9 & 33,33 \\
Ignorado & 6 & 22,22 & 4 & 14,80 \\
\hline
\end{tabular}

A maioria dos cirurgiões-dentistas e dos auxiliares em saúde bucal acredita que os acidentes foram causados por desatenção (70\% e 38,5\%, respectivamente). Os auxiliares em saúde bucal também atribuíram os acidentes a pressa $(15,4 \%)$ e cansaço $(15,4 \%)$. Dentre as citadas como "outras causas", relatou-se tamanho de luva inadequado, causalidade, um caso de respingo na mucosa ocular e outro onde o instrumental estava embrulhado na toalha. Os acidentes atribuídos a "outras causas" corresponderam a aproximadamente $30 \%$ das respostas dos participantes.

Os objetos que mais causaram os acidentes em cirurgiões-dentistas foram as agulhas (40\%), seguidos dos instrumentais (30\%), sendo que para os auxiliares em saúde bucal o instrumental foi o objeto causador mais frequente do acidente $(84,6 \%)$, seguido das agulhas $(15,4 \%)$.

O Gráfico 1 apresenta os tipos de lesões dos acidentes com exposição a material biológico de acordo com os dados obtidos nas CAT do Núcleo de Agravos Notificáveis da Secretaria Municipal de Saúde de Natal. As CAT de 2008 apresentaram 19 (70,37\%) lesões percutâneas, 5 (18,51\%) lesões em pele íntegra, $1(3,70 \%)$ em mucosa e $2(7,40 \%)$ foram ignoradas. Em 2009, dos 27 acidentes registrados, 23 (85,18\%) foram lesões percutâneas e $4(14,81 \%)$ lesões em pele íntegra.

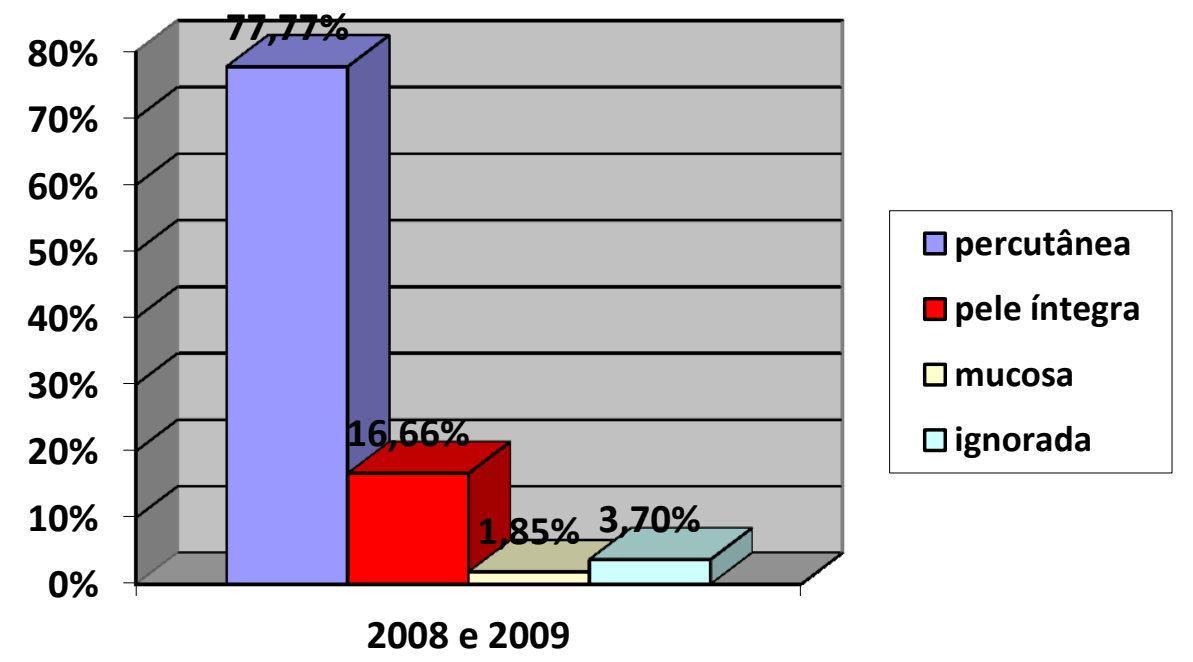

Gráfico 1: Tipos de lesão dos acidentes com exposição a material biológico em Odontologia em 2008 e 2009. Natal-RN. 2010. 
A maior parte dos acidentes ocorridos com auxiliares em saúde bucal aconteceu à tarde $(61,5 \%)$, e com os cirurgiões-dentistas, pela manhã (70\%). Com relação às horas trabalhadas no dia do acidente, $53,8 \%$ dos auxiliares em saúde bucal e $70 \%$ dos cirurgiões-dentistas haviam trabalhado até quatro horas.

Observou-se que $90 \%$ dos cirurgiões-dentistas e 100\% dos auxiliares em saúde bucal consideraram suas mãos como a parte do corpo mais atingida pelos acidentes. Em 76,9\% dos acidentes ocorridos com auxiliares em saúde bucal e em $90 \%$ dos acidentes ocorridos com cirurgiões-dentistas os profissionais usavam os seguintes EPI: luva, máscara, gorro, óculos e jaleco.

Os dados da pesquisa revelaram que $94,2 \%$ dos auxiliares em saúde bucal e $79,7 \%$ dos cirurgiõesdentistas acreditam que os acidentes com exposição a material biológico devem obrigatoriamente ser notificados e dentre as doenças que mais preocupam os profissionais nesse tipo de acidente estão a SIDA e a hepatite C. A SIDA preocupa 100\% dos cirurgiões-dentistas e $98,6 \%$ dos auxiliares em saúde bucal. A hepatite $C$ foi a segunda doença citada como mais preocupante entre cirurgiões-dentistas e auxiliares em saúde bucal (81,5\% e 74,3\% respectivamente). Dentre outras doenças que preocupam os profissionais da equipe de saúde bucal estão a Gripe H1N1, outras doenças sexualmente transmissíveis (sífilis e outras), conjuntivite e cegueira.

A portaria n $777 /$ GM que dispõe sobre os procedimentos técnicos para a notificação compulsória de agravos à saúde do trabalhador regulamenta a notificação compulsória de agravos à saúde do trabalhador acidentes e doenças relacionados ao trabalho. Considerando que a gravidade do quadro de saúde dos trabalhadores brasileiros está expressa, a portaria estabelece que, dentre outros, são agravos de notificação compulsória os "Acidentes com Exposição a Material Biológico". O Instrumento de Notificação Compulsória é a Ficha de Notificação, padronizada pelo Ministério da Saúde, segundo o fluxo do Sistema de Informação de Agravos de Notificação (SINAN)1,2,12.

No Núcleo de Agravos Notificáveis foram notificados 4 acidentes em cirurgiões-dentistas e 3 acidentes em auxiliares de saúde bucal. Levando-se em consideração que na coleta direta feita junto aos profissionais houve relato de 10 acidentes em cirurgiões-dentistas e 13 em auxiliares em saúde bucal no período estudado, obteve-se um percentual de notificações bem abaixo do real de apenas $40 \%$ e $15,3 \%$ respectivamente.

Tabela 3: Informações contidas no formulário da CAT quanto ao conhecimento da fonte relacionada ao acidente. Natal-RN, 2010.

\begin{tabular}{ccccccc}
\hline & \multicolumn{2}{c}{$\mathbf{2 0 0 8}$} & \multicolumn{2}{c}{$\mathbf{2 0 0 9}$} & \multicolumn{2}{c}{$\mathbf{2 0 0 8}$ e 2009 } \\
& $\mathbf{N}$ & $\mathbf{\%}$ & $\mathbf{N}$ & $\mathbf{\%}$ & $\mathbf{N}$ & $\mathbf{\%}$ \\
\hline Sim & 11 & 40,74 & 7 & 25,92 & 18 & 33,33 \\
Não & 5 & 18,51 & 6 & 22,22 & 11 & 9,25 \\
Ignorado & 10 & 37,03 & 14 & 51,85 & 24 & 44,44 \\
\hline
\end{tabular}




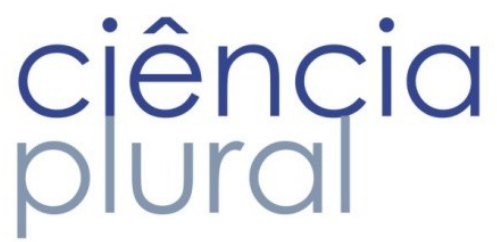

A Tabela 3 registra as informações associadas ao conhecimento da fonte relacionada ao acidente. Quase metade das CAT ignoravam este dado, demonstrando descaso com dados essenciais para decisões relacionadas à conduta pós-acidente. Os dados relativos à fonte relacionada ao acidente permitem que 0 médico que assiste ao acidentado decida qual caminho tomar em relação a medidas profiláticas.

\section{Discussão}

Riscos ocupacionais podem e devem ser minimizados favorecendo a diminuição dos acidentes de trabalho em diversas categorias profissionais. A partir do reconhecimento destes riscos, da identificação dos problemas e da estimativa da prevalência destes acidentes, medidas preventivas e programas intervencionistas

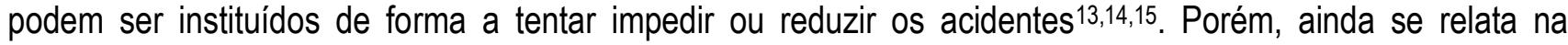
literatura que há escassez de conhecimento, ausência ou falta de padronização nos sistemas de informações que permitam conhecer a real magnitude dos acidentes de trabalho com exposição a material biológico em cirurgiões-dentistas e auxiliares em saúde bucal9,10, sendo este um dos fatos que motivaram esta pesquisa.

Dentre os acidentes registrados na literatura, os que envolvem o manuseio de instrumentos perfurocortantes são relatados como muito prevalentes e preocupantes. Os profissionais de saúde compõem as categorias mais atingidas por este tipo de acidente ${ }^{6,7,15}$. As pesquisas são constantes em relatar que apesar de haver quantidade relevante de estudos sobre o tema, a prevalência deste tipo de agravo ainda é considerada alarmante. São motivo de preocupações para trabalhadores e instituições no Brasil e no mundo 8,16.

Embora os acidentes de trabalho com exposição a material biológico ocorram com grande frequência nos diversos profissionais que atuam em ambiente hospitalar 7,12,19 os autores enfatizam a importância de pesquisas que estimem a prevalência de acidentes de trabalho no serviço público abrangendo unidades básicas de saúde, que é o caso desta pesquisa, uma vez que já foi constatada a importância do conhecimento destes agravos e a necessidade de implantação de programas no serviço público que previnam estes acidentes e sensibilizem os profissionais sobre a gravidade dos mesmos ${ }^{15,16}$.

Diversas doenças podem surgir como consequência de acidentes levando a impactos na vida da vítima, interferindo nos aspectos familiares, psicológicos e sociais ${ }^{5}$. Com relação aos acidentes com exposição a material biológico as doenças que mais preocuparam os cirurgiões-dentistas e auxiliares em saúde bucal nesta pesquisa foram a SIDA e a hepatite C, corroborando com os achados na literatura 17,18. Embora a SIDA tenha surgido por volta da década de 80 , ainda hoje persistem as preocupações referentes às formas de transmissão da mesma ${ }^{18}$. Outras doenças aludidas na literatura também foram citadas pelos profissionais entrevistados nesta pesquisa como a síflis dentre outras doenças sexualmente transmissíveis, conjuntivite e cegueira. Atualmente, a epidemia da influenza a H1N1 trouxe preocupações também aos profissionais da odontologia, que citaram como uma das doenças que os atemorizam ao sofrerem acidentes de trabalho com exposição a material biológico.

Profissionais da área de saúde devem receber vacina contra a hepatite B, sarampo, parotidite, rubéola e tétano, ainda que o risco de contrair tétano seja praticamente nulo. Também é fundamental tratar fluidos corporais, como a saliva, exsudato e sangue como potencialmente contaminados. Essa preocupação deve ser profissional e pública, empregando as precauções universais e as barreiras de proteção para auxiliar no controle da infecção cruzada ${ }^{19}$. 


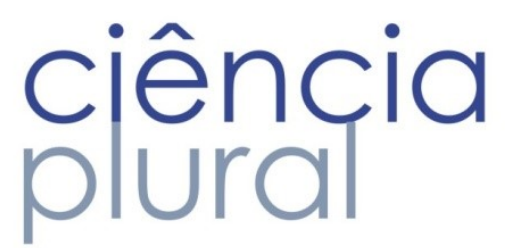

A prevalência de acidentes de trabalho com exposição a material biológico em cirurgiões-dentistas e auxiliares na presente pesquisa foi de 12,8\% para cirurgiões-dentistas e 18,3\% para auxiliares em saúde bucal. A literatura relata este tipo de acidente como frequente, mas os percentuais variam em larga faixa, dependendo das características da pesquisa. Estudos relatam muitas vezes, apenas os registros dos acidentes a órgãos de vigilância sanitária através de documentos como as CAT, prontuários de atendimento em serviços de saúde, ou estatísticas oficiais ${ }^{7,18,20}$ e às vezes apenas 0 relato do próprio acidentado frequentemente através de questionários ou entrevistas ${ }^{19,20,21}$. Embora haja grande quantidade de estudos sobre acidentes de trabalho com exposição a material biológico, este estudo apresentou uma característica inovadora: onde tanto os profissionais participantes da pesquisa relataram se sofreram acidentes de trabalho através do questionário que responderam, como a consulta junto às CAT, que também foi fonte de informações de prevalência dos acidentes ocorridos na população do estudo, percebendo-se de início uma divergência entre um dado e outro.

Alguns percentuais descritos na literatura de acidentes em cirurgiões-dentistas variam de $39 \%{ }^{9}, 74 \% 21$, até a $94,5 \% 9$. Poucos estudos referem-se a outras categorias da odontologia, mas foram encontrados percentuais na literatura até $80,4 \%$ de acidentes em auxiliares em saúde bucal ${ }^{9}$. Também é relatada na literatura a prevalência de acidentes com exposição a material biológico em outros países como a Jordânia, 66,5\%13, Afeganistão, 62,6\% ${ }^{19}$, Alemanha, 61,7\% em cirurgiões-dentistas e 71,7\% em auxiliares em saúde bucal 22 .

A pesquisa que mais se aproxima da metodologia deste estudo foi a de Garcia \& Blank $(2006)^{6}$, que pesquisou a prevalência de acidentes com exposição a material biológico em cirurgiões-dentistas e auxiliares em saúde bucal em Santa Catarina, Brasil, onde houve uma prevalência aproximada de 39\% para cirurgiõesdentistas e auxiliares em saúde bucal no último ano de trabalho. Os dados de prevalência de acidentes encontrados na pesquisa em Natal-RN demonstram uma prevalência inferior àquela encontrada na literatura ${ }^{6}$.

Algumas pesquisas não encontraram associações estatisticamente significativas entre a ocorrência de acidentes e idade ou gênero ${ }^{15}$. Neste estudo optou-se por não realizar associações entre as variáveis gênero e acidentes uma vez que se observaram grandes diferenças entre o número de profissionais do sexo masculino $\mathrm{e}$ feminino e também em relação às idades dos profissionais, ou seja, não havia homogeneidade da amostra.

O material biológico ao qual os profissionais foram mais expostos no momento do acidente foi o sangue, corroborando com estudos que pesquisaram o tipo de fluido envolvido nos acidentes ${ }^{7}$. 0 estudo de Garcia \& Blank $(2006)^{6}$ obteve resultados diferentes pois a maioria dos profissionais acidentados relataram que a saliva sem sangue visível foi o material biológico envolvido nos acidentes. Acredita-se que na pesquisa citada, os profissionais acidentados consideraram os respingos como exposições ocupacionais, enquanto que na presente pesquisa é possível que os cirurgiões-dentistas e auxiliares em saúde bucal não tenham 0 conhecimento, ou apenas negligenciaram o fato que respingos em mucosas, como a ocular e nasal, por exemplo, também podem ser considerados acidentes com exposição a material biológico. Embora alguns estudos não relatem diretamente qual o material biológico mais frequente nas exposições, termos como "acidentes perfuro-cortantes" ou "injúrias percutâneas" são bastante utilizados, propondo que o material biológico envolvido no acidente seria o sangue 8,12,14,19,21.

Os dados sobre os acidentes relatados neste estudo sugeriram que procedimentos relacionados a cirurgia e periodontia estão mais relacionados a acidentes de trabalho com exposição a material biológico, corroborando com dados de outras pesquisas que apontam procedimentos como cirurgias odontológicas e suturas, por exemplo, onde o uso de instrumentos cortantes é frequente, como os procedimentos que estavam sendo realizados no momento do acidente 7 . Alguns estudos apresentaram dados que se referem a procedimentos de dentística como os que estão envolvidos nos acidentes ${ }^{9}$. 


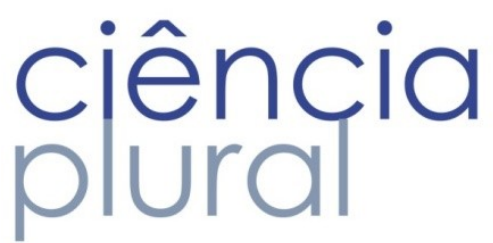

As causas dos acidentes de trabalho com exposição a material biológico ou com material perfurocortante nos profissionais da Odontologia são diversas, como pressa, cansaço, nervosismo, repetição das ações ou volume de trabalho, estresse, despreparo ou falta de atenção quanto às medidas de precauções universais, além da desatenção ou falha pessoal7,18,21,22. Os autores ainda citam que acidentes com material perfuro-cortante, usados rotineiramente na Odontologia, são bastante comuns quando há descarte em locais inadequados ou em recipientes cheios e/ou abarrotados, quando há transporte ou manipulação de agulhas desprotegidas e desconexão da agulha da seringa, mas a principal causa deste tipo de acidente ainda é 0 reencape das agulhas $5,7,15,19$.

Neste estudo, aproximadamente $70 \%$ dos acidentes em cirurgiões-dentistas e quase $40 \%$ dos acidentes em auxiliares em saúde bucal foram causados por desatenção, sendo corroborado por outros estudos ${ }^{21,22}$. Além disso, outras causas como EPI inadequado e/ou incompleto e respingos em mucosa ocular também foram considerados causas dos acidentes pelos participantes da pesquisa, assim como em outras pesquisas ${ }^{12,16,18 .}$

Os objetos utilizados no momento dos acidentes e relacionados aos mesmos como causadores nesta pesquisa foram agulhas ocas, agulhas de sutura e instrumentais, sendo os primeiros mais mencionados pelos cirurgiões-dentistas e os últimos pelos auxiliares em saúde bucal. Isto provavelmente se deve ao fato de cirurgiões-dentistas utilizarem as agulhas em procedimentos anestésicos e também podem ser em decorrência de reencape das mesmas. Com relação aos instrumentais, imagina-se que os auxiliares em saúde bucal relacione-os aos acidentes devido à lavagem dos mesmos ser realizada por estes profissionais com muito mais frequência do que pelos cirurgiões-dentistas. Estes dados são corroborados pela maioria dos estudos encontrados na literatura que, devido ao uso rotineiro de agulhas e instrumentais na clínica diária da Odontologia, tais objetos perfuro-cortantes tornam-se frequentemente relacionados aos acidentes 14,15,19,20,22.

Em outro estudo, a incidência de injúrias decorrentes de agulhas e as suas notificações foram pesquisadas através de um estudo de corte seccional entre dentistas do Norte da Jordânia. 0 estudo incluiu 170 clínicos gerais, dos quais, $113(66,5 \%)$ se acidentaram nos 12 meses anteriores à pesquisa. As injúrias foram associadas significativamente a mais idade e maior número de pacientes atendidos diariamente. Daqueles que se acidentaram $77,9 \%$ não registraram o acidente e as razões para isso foram: 0 acidente aconteceu antes do uso no paciente $(41,2 \%)$, desconhecimento do risco $(20,8 \%)$, o profissional estava ocupado $(25 \%)$ e insatisfação com os procedimentos pós-acidente (13\%). Neste estudo, $12,4 \%$ dos acidentes ocorreram durante o reencape e antes da agulha ser usada e $11,5 \%$ durante a abertura da embalagem da agulha. Este tipo de injúria é de menor risco para o cirurgião-dentista, mas se a agulha for usada posteriormente, torna-se um risco potencial para o paciente $^{3}$.

Alguns estudos citam outros objetos como brocas, bisturis e limas de endodontia como relacionados aos acidentes, mas durante esta pesquisa não houve frequência de relatos de acidentes com tais objetos $7,8,9,20$. Os acidentes com exposição a material biológico podem ainda ser causados por mordeduras, por respingos ou mesmo contato na pele intacta, mas observou-se que os profissionais relacionam acidentes com exposição a material biológico com maior frequência a lesões percutâneas, ou seja, aquelas que perfuram ou cortam a pele por meio de instrumentos como as agulhas e os instrumentais. Torna-se importante ressaltar em programas de educação continuada a sensibilização dos profissionais no que se refere a estes tipos de acidentes, que embora aparentemente sejam de menor importância, podem levar a consequências graves e doenças com sequelas, e mesmo podem ser motivo de infecção cruzada dentro do consultório odontológico. Toda a equipe de saúde bucal tem obrigação de não negligenciar tais acidentes de trabalho uma vez que é responsável pela sua própria saúde, mas também se torna responsável por não provocar danos àqueles que frequentam 0 ambiente odontológico, sejam pacientes ou colegas. 


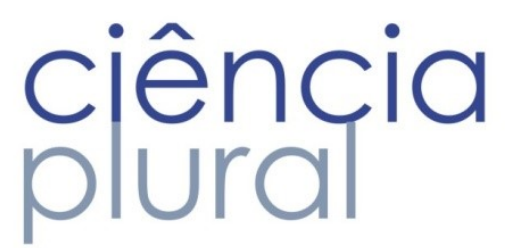

As investigações das circunstâncias dos acidentes de trabalho são um meio eficaz para o planejamento dos riscos ocupacionais, como as observações de aspectos relacionados aos acidentes como horários e setores que mais ocorrem, os profissionais mais expostos, a área do corpo mais atingida e a profilaxia adotada nestes casos. É importante planejar e elaborar estratégias que possam prevenir a ocorrência de novos casos de acidentes, resultando em segurança para os trabalhadores da área em questão ${ }^{18}$.

Chiodi et al $(2007)^{4}$, investigaram a ocorrência de acidentes de trabalho com exposição a material biológico entre trabalhadores da saúde atuantes em Unidades de Saúde Pública de Ribeirão Preto, São Paulo. Para tanto, foram utilizadas as Comunicações de Acidente de Trabalho do ano de 2005 disponíveis na Divisão de Medicina e Segurança do Trabalho da Prefeitura do Município. Dos 155 acidentes de trabalho registrados, em $40 \%$ houve exposição a material biológico. Os autores relatam que este número é característico de subnotificação uma vez que há um grande número de trabalhadores expostos. Dos 362 cirurgiões-dentistas registrados na Secretaria Municipal de Saúde, 11 registraram algum acidente de trabalho com exposição a material biológio, sendo a segunda categoria mais atingida pelos acidentes na pesquisa (17,7\%). As cirurgias odontológicas foram responsáveis por $17,7 \%$ dos acidentes de trabalho e as suturas por $11,29 \%$, enquanto 0 descarte de material perfuro-cortante correspondeu a $9,68 \%$ dos acidentes de trabalho ${ }^{4}$.

É possível que acidentes ocorram com maior frequência em determinados períodos do dia ou mesmo que a quantidade de horas trabalhadas no dia do acidente influencie a sua ocorrência. Neste estudo observouse que a maior parte dos acidentes ocorridos em auxiliares em saúde bucal aconteceu à tarde $(61,5 \%)$, e nos cirurgiões-dentistas, pela manhã (70\%). Quanto mais horas o profissional houver trabalhado, maiores as chances de ele ter atendido mais pacientes, de ter realizado mais procedimentos e, portanto, acreditar-se-ia que haveria maior probabilidade de acontecer acidentes. Porém, neste estudo observou-se que dos profissionais acidentados, 60,83\% acidentaram-se após haver trabalhado até 4 horas, sendo que dados semelhantes foram encontrados em outros estudos ${ }^{9}$.

No estudo de Medeiros Junior (2005) $)^{9}$, o autor identificou os elementos representacionais sobre os acidentes ocupacionais em saúde e investigou os tipos mais frequentes em um hospital universitário de Natal. As conclusões mostraram que os acidentes perfuro-cortantes foram os que mais ocorreram $(61,8 \%)$ e que as representações dos sujeitos se entrelaçam constituindo um discurso tradicional sobre acidentes de trabalho. Constatou ainda que os trabalhadores satisfeitos com o trabalho sofreram proporcionalmente 2,55\% menos acidentes. Apenas $38,8 \%$ dos trabalhadores acidentados usavam EPI no momento do acidente mostrando a necessidade de reforço de motivação para utilização dos equipamentos, além da necessidade de disponibilização dos mesmos nos locais de trabalho. $O$ autor ressalta a importância de priorizar as questões relacionadas aos acidentes de trabalho e a necessidade de envolver ações destinadas a construção de um sistema local de informações que seja capaz de captar de maneira mais adequada a ocorrência destes fenômenos e também de analisar as fontes de risco para a elaboração de um plano de controle e avaliação do impacto destas avaliações ${ }^{9}$.

Garcia \& Blank (2006) ${ }^{6}$ não encontraram associações entre a ocorrência de exposições ocupacionais e as variáveis: horas de trabalho por dia, dias de trabalho por semana, horas de trabalho por semana e frequência de pausas durante o trabalho. Outro estudo, realizado em um hospital público do Peru constatou que, na população estudada, $57,6 \%$ dos acidentes aconteceram durante as primeiras quatro horas de trabalho, porém, quase $60 \%$ deles aconteceram após o meio-dia ${ }^{14}$. Portanto, sugere-se que mais estudos sejam realizados levando em considerações as variáveis relacionadas a horas de trabalho dos profissionais, bem como as possíveis associações que possam existir entre os acidentes e os turnos trabalhados.

Acidentes de trabalho com exposição a material biológico podem acometer diversas partes do corpo como membros superiores, olhos, face e pés. Neste estudo, aproximadamente $91 \%$ dos profissionais relataram 


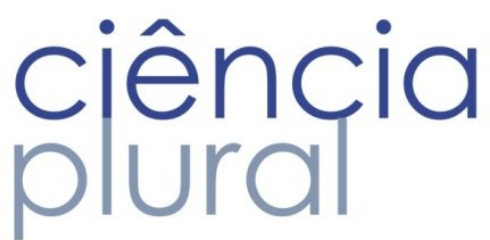

que as suas mãos foi a parte do corpo mais atingida pelos acidentes. Os dados desta pesquisa são reiterados por dados de outros estudos realizados, onde os acidentes de trabalho envolveram membros superiores, principalmente mãos e dedos 7,9 . Este dado se justifica pelo fato do profissional da odontologia utilizar-se essencialmente dos seus membros superiores durante os procedimentos clínicos. A área de maior exposição dos profissionais é a face e os membros superiores, proporcionando maior probabilidade de ocorrência de acidentes, principalmente nas mãos e nos dedos.

Quanto à notificação, somente $16,7 \%$ dentistas que sofreram acidentes efetivaram o registro por meio da Comunicação de Acidente de Trabalho (CAT). Esse resultado indica alto nível de subnotificação de acidentes, resultado que pode significar que à CAT não é dada a devida importância, fato que resulta em baixo índice de ocorrência das estatísticas relativas a acidentes de trabalho, em especial, no campo da Odontologia.

Algumas pesquisas relacionam a possibilidade maior de ocorrência de acidentes com 0 uso de Equipamento de Proteção Individual-EPI incompleto. A pesquisa de Marchado-Carvalhais(2008)12 realizada em estudantes de odontologia de uma universidade pública do Brasil constatou através de uma análise estatística bivariada na população estudada uma associação estatisticamente significativa entre acidentes ocupacionais com exposição a sangue e uso incompleto de EPI ${ }^{12}$. Neste estudo, aproximadamente $82 \%$ dos acidentados relataram que usavam EPI completo no momento do acidente, sugerindo que não houve relação entre os acidentes e o uso de EPI. Este dado é similar ao encontrado no estudo de Teixeira, et al $(2008)^{16}$ onde mesmo com o uso rotineiro de EPI, observou-se uma prevalência de $73 \%$ de acidentes nos cirurgiões-dentistas.

A maioria dos participantes da pesquisa $(79,7, \%$ de cirurgiões-dentistas e $94,2 \%$ de auxiliares em saúde bucal) afirmou que os acidentes de trabalho com exposição a material biológico devem obrigatoriamente ser notificados, concordando com o disposto na Portaria $n^{0} 777 / \mathrm{GM}^{2}$, porém quando se tratou de colocar em prática o que requer a legislação, houve negligência, uma vez que todos os profissionais que sofreram acidentes afirmaram que não houve registro dos mesmos. Este registro pode não ter ocorrido porque o próprio profissional não procurou pronto-atendimento ou porque no local do pronto-atendimento não foi realizada a comunicação do acidente de trabalho.

Portanto na presente pesquisa constatou-se uma subnotificação dos acidentes de trabalho com exposição a material biológico. Dos acidentes ocorridos relatados pelos próprios profissionais nos questionários, foram registrados no NAN apenas $40 \%$ e 15,3\% dos acidentes em cirurgiões-dentistas e auxiliares em saúde bucal, respectivamente. Suspeita-se que além da subnotificação dos acidentes relatados nos questionários, mais acidentes tenham ocorrido nesta população, inclusive acidentes de outras naturezas como os decorrentes de riscos mecânicos, químicos, ergonômicos de trajeto e as doenças relacionadas ao trabalho.

É oportuno ressaltar a obrigatoriedade constante na Portaria $n^{0} 777 / \mathrm{GM}$ que afirma que acidentes de trabalho com exposição a material biológico são agravos de notificação compulsória ${ }^{2}$. A portaria $n^{0} 777 / G M$ dispõe sobre os procedimentos técnicos para a notificação compulsória de agravos à saúde do trabalhador e regulamenta a notificação compulsória de agravos à saúde do trabalhador - acidentes e doenças relacionados ao trabalho. Considerando que a gravidade do quadro de saúde dos trabalhadores brasileiros está expressa, a portaria estabelece que, dentre outros, são agravos de notificação compulsória os Acidentes com Exposição a Material Biológico. O Instrumento de Notificação Compulsória é a Ficha de Notificação, padronizada pelo Ministério da Saúde, segundo o fluxo do Sistema de Informação de Agravos de Notificação (SINAN) ${ }^{1,2}$.

Alguns estudos relatam números alarmantes de acidentes de outras natureza acontecidos em trabalhadores de saúde, como 0 estudo de Miranzi et al $(2008)^{13}$ que constatou o registro de um percentual de 


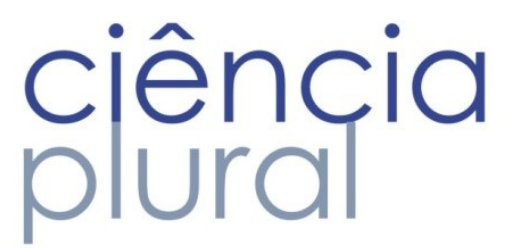

$24 \%$ de acidentes decorrentes de esforço físico, queda, entorse, lombalgia, cervicalgia e impacto e $7 \%$ de acidentes de trajeto ${ }^{13}$.

Por fim, é oportuno ressaltar que o Ministério da Saúde destaca o fato do acidente ser um evento que pode ser previsível e previnível, independente da situação empregatícia do acidentado. Estudo que delineam as características dos acidentes procurando compreendê-los nos seus detalhes e buscam sensibilizar os profissionais quanto à sua prevenção, colaboram na busca pela previsibilidade e prevenção dos acidentes. Além de compreender os acidentes, é importante conhecer todas as questões relativas a culpabilidade relacionadas ao trabalhador nos acidentes além dos impactos que estes acidentes podem trazer para a vítima e sua família. É importante que os serviços públicos assumam seu papel preventivo e intervencionista nas questões relacionadas aos acidentes de trabalho, refletindo sobre o processo de trabalho nas instituições públicas e a possibilidade de sua reorganização reduzindo os riscos ocupacionais e promovendo qualidade de trabalho e consequentemente de vida aos profissionais ${ }^{5,18}$. Este estudo buscou não apenas quantificar $\mathrm{e}$ caracterizar os acidentes de trabalho com exposição a material biológico, mas principalmente contribuir nas reflexões que levam a melhorias na qualidade de vida dos trabalhadores.

\section{Conclusões}

A prevalência de acidentes de trabalho com exposição a material biológico foi em torno de $13 \%$ (cirurgiões-dentistas) e 18\% (auxiliares em saúde bucal). Apesar de serem os causadores da maioria dos acidentes pouca atenção é dispensada aos acidentes com instrumentais perfuro-cortantes, existindo uma significativa subnotificação. A preocupação em preveni-los deve ser uma constante, em função das graves consequências que acometem os trabalhadores expostos a esses acidentes, sinalizando para que a notificação faça parte da conduta da equipe de saúde.

A maioria dos acidentes de trabalho envolveu sangue, e a desatenção foi o fator primordial relacionado à sua causa. A atitude dos cirurgiões-dentistas e auxiliares em saúde bucal de nem sempre tomarem medidas após 0 acidente é preocupante, especialmente porque a exposição pode ser minimizada na medida em que sejam adotadas boas práticas de trabalho e organização profissional na atividade de atendimento odontológico tais como: uso de equipamentos de proteção coletiva e individual, adequação das instalações físicas aos preceitos da biossegurança e capacitação continuada dos cirurgiões-dentistas e auxiliares de saúde bucal.

Portanto, os acidentes ocupacionais devem ser prevenidos e analisados no ambiente profissional por meio da divulgação de métodos que ressaltem a importância da biossegurança, o manuseio correto dos instrumentos e materiais biológicos, a imunização completa e protocolos de como proceder em casos de acidentes.

Sugere-se que as instituições públicas colaborem no sentido de sensibilizar e educar os trabalhadores de saúde quanto à segurança no trabalho através de atividades educativas que visem qualidade nas condições de trabalho com consequente aumento da qualidade de vida. Sugere-se também maior divulgação da importância de notificação acidentes de trabalho com exposição a material biológico, uma vez que estando registrados possibilitam a elaboração e implementação de políticas públicas, visando maior segurança e qualidade de vida do trabalhador da saúde. 


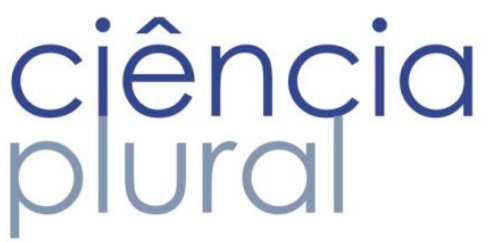

Referências

1. Brasil. Portaria $n^{0} 777 / G M$ de $28 / 04 / 04$. Dispõe sobre os procedimentos técnicos para a notificação compulsória de agravos à saúde do trabalhador em rede de serviços sentinela específica, no Sistema Único de Saúde - SUS.

2. Brasil. Ministério da Saúde. Secretaria de Atenção à Saúde. Departamento de Ações Programáticas Estratégicas. Notificação de Acidentes de Trabalho fatais, graves e com crianças e adolescentes. Brasília, 2006. Disponível em: http://bvms.saude.gov.br/bvs/publicacoes/protocolo_not_acidentes_trab.pdf

3. Al-Khatib IA, Ishtayeh M, Barghouty H, Akkawi B. Dentists' perceptions of occupational hazards and preventive measures in East Jerusalem. Eastern Mediterranean Health Journal. 2006; 12(1/2):153-160.

4. Chiodi MB; Marziale MHP; Robazzi MLCC. Acidentes de trabalho com material biológico entre trabalhadores de unidades de saúde pública. Rev Latino-am Enfermagem. 2007;15(4):123-129.

5. Garbin AJI, Presta AA, Garbin CAS, Lima DC. Ocurrencia de accidentes ocupacionales y conducta em la práctica odontológica. Revista Cubana de Salud y Trabajo. 2006;7(1-2):29-33

6. Garcia LP, Blank VLG. Prevalência de exposições ocupacionais de cirurgiões-dentistas e auxiliares de consultório dentário a material biológico. Cad. Saúde Pública. 2006;22(1):97-108.

7. Giorgis RS, Primo LS, Edom FT, Oliveira TC, Hosni E. Acidentes com material biológico no dia-a-dia da clínica odontológica: como proceder?. RBO. 2003;60(4):247-251.

8. Gonçalves JA. Acidente de trabalho entre a equipe assistencial multiprofissional uma avaliação da subnotificação [dissertação]. Belo Horizonte: Universidade Federal de Minas Gerais; 2007.

9. Medeiros Júnior, A. Representação Social sobre o acidente de trabalho na área de saúde. [Tese]. Natal: Universidade Federal do Rio Grande do Norte: Programa de Pós Graduação em Ciências da Saúde; 2005.

10. Khader $Y$, Burgan S, Amarin Z. Self-reported needle-stick injuries among dentists in north Jordan. Eastern Mediterranean Health Journal. 2009;15(1):185-189.

11. Machado-Carvalhais HPM, Martins TCPM, Ramos-Jorge ML, Magela-Machado D, Paiva SM, Pordeus IA. Management of occupational bloodborne exposure in a dental teaching environment. J Dent Educ. 2007;71(10):1348-1355.

12. Machado-Carvalhais HPM, Ramos-Jorge ML, Auad SM, Martins LHPM, Paiva SM, Pordeus IA. Occupational exposure to potentially infectious biological material in a dental teaching environment. J Dent Educ. 2008;72(10):1201-1208. 


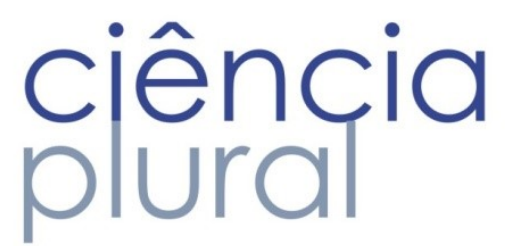

13. Miranzi SSC, Gaspar AACS, Iwamoto HH, Miranzi MAS, Dziabas DC. Acidentes de trabalho entre os trabalhadores de uma universidade pública. Rev. bras. Saúde ocup. 2008;33(118):40-47.

14. Salehi AS, Garner P. Occupational injury history and universal precautions awareness: a survey in Kabul hospital staff. BioMed Central. 2010;10(19):1-4.

15. Shah SM, Merchant AT, Dosman JA. Percutaneous injuries among dental professionals in Washington State. BMC Public Health. 2006;6:269.

16. Teixeira CS, Pasternak-Júnior B, Silva-sousa YT, Correa-Silva SR. Medidas de prevenção pré e pósexposição a acidentes perfuro cortantes na prática odontológica. Rev. Odonto ciênc. 2008;23(1):10-14.

17. Orestes-Cardoso SM, Farias ABL, Pereira MRG, Orestes-Cardoso AJ, Cunha Júnior IF. Acidentes perfuro cortantes: prevalência e medidas profiláticas em alunos de odontologia. Rev. bras. Saúde ocup., São Paulo, 34 (119): 06-14, 2009

18. Wicker S, Rabenau HF. Occupational exposures to bloodborne viruses among German dental professionals and students in a clinical setting. Int Arch Occup Environ Health.2010; 83:77-83.

19. Bragança DPP, FernandeS MM, Sassi C, Francesquini Júnior L, Daruge Júnior E. Condutas do cirurgiãodentista frente a acidentes biológicos. Odonto 2010;18(35):37-44.

20. Andrade RRA, Almeida RAC, Sampaio GC, Pereira JRD, Andrade ESS. Ocorrência de acidentes com instrumentais pérfuro-cortantes em clínica odontológica na cidade do Recife-Pernambuco-Estudo-piloto. Rev. Cir. Traumatol. Buco-Maxilo-Fac., Camaragibe 2013, 13(2): 87-100.

21. Gabler IG, Freitas NA, Boa Morte NCGR, David IA, Salim M, Prado R. Prevenção e ocorrência de acidentes com materiais perfuro cortantes entre os profissionais da área odontológica da cidade de Vila Velha/ES. Rev. bras. odontol., Rio de Janeiro, 69(2):174-9, jul./dez. 2012

22. Nascimento LS, Assunção LRS, Silva Júnior NG, Pedreira EN, Silva RLC. Acidentes com perfuro cortantes na Faculdade de Odontologia da UFPA: visualização de um Cenário. Rev Odontol Bras Central 2012; 21(56):463-467. 